\title{
Hippocampal atrophy correlates with severe cognitive impairment in elderly patients with suspected normal pressure hydrocephalus
}

J Golomb, M J de Leon, A E George, A Kluger, A Convit, H Rusinek, S de Santi, A Litt, $S$ H Foo, S H Ferris

\begin{abstract}
Measurements of hippocampal formation atrophy using MRI have been useful in distinguishing demented patients with a diagnosis of probable Alzheimer's disease from cognitively normal controls. To determine whether there is a similar relationship between hippocampal size and dementia in elderly patients suspected of normal pressure hydrocephalus (NPH), the authors obtained mini-mental status examination (MMSE) scores and MRI measurements of hippocampal size and CSF volume on 16 elderly patients whose severe ventriculomegaly and unexplained gait impairment made NPH a probable diagnosis. Hippocampal size correlated strongly with MMSE score ( $r$ $=0.75, p<0.001)$; no significant MMSE correlation was found for ventricular CSF volume or extraventricular/ventricular CSF ratio. It was concluded that hippocampal atrophy is associated with severe cognitive dysfunction in many elderly patients with a diagnosis of NPH. As a hypothesis for further investigation, the detection of such atrophy may help identify cases where the presence of a pathology of Alzheimer's disease complicates the diagnosis of NPH.
\end{abstract}

$(\Im$ Neurol Neurosurg Psychiatry 1994;57:590-593)

Aging and Dementia Research Center, New York University Medical Center, New York, USA Department of Neurology

$\mathrm{J}$ Golomb

S H Foo

Department of

Psychiatry

M J de Leon

A Kluger

A Convit

$S$ de Santi

S H Ferris

Department of

Radiology

A E George

H Rusinek

A Litt

Correspondence to:

Dr James Golomb, Aging

Dr James Golomb, Aging
and Dementia Research

and Dementia Research

Center, New York University
School of Medicine, HN314,

550 First Avenue, New York,

550 First Avenue, New York America.

Received 22 April 1993

and in revised form

28 July 1993.

Accepted 30 September 1993

pressure hydrocephalus (NPH) often difficult to diagnose in older patients. When elderly individuals exhibit dementia, unexplained gait dysfunction, and excessive ventriculomegaly as their only significant neuroradiographic abnormality, it may be unclear whether NPH, a primary degenerative illness, or a combination of these two processes, may be the correct diagnosis. ${ }^{1-4}$ For example, the extremely high prevalence of Alzheimer's disease as a cause of non-vascular dementia in the elderly increases the likelihood that this condition is present in many patients with clinical and radiographic findings consistent with a diagnosis of NPH. Conventional imaging strategies for identifying ex vacuo ventriculomegaly caused by degenerative brain disease have emphasised appraisals of sulcal prominence. ${ }^{5}$ Such assessments may be misleading when applied to older patients for whom the possibility of coexistent NPH and degenerative disease exists. If Alzheimer's disease occurs in a patient with NPH, the pattern of sulcal widening which is characteristic of the first condition may not be apparent. These considerations emphasise the need to explore other brain characteristics of potential specific value in diagnosing neurodegenerative illness.

Recent neuroimaging studies demonstrate that atrophy of the hippocampal formation is a characteristic of Alzheimer's disease. ${ }^{67}$ The authors speculate that this observation may have implications for identifying cases where Alzheimer's disease complicates the diagnosis of NPH. It is recognised that cognitive abnormalities are not always evident in NPH and gait impairment may be the only symptom..$^{4}$ If Alzheimer's disease explains the dementia of many elderly patients with ataxia and ventriculomegaly consistent with $\mathrm{NPH}$, such patients would be expected to exhibit hippocampal atrophy on clinical radiography in excess of that in similar individuals with mild or no evidence of intellectual dysfunction. In a study designed to test this hypothesis, the authors examined the relationship between hippocampal size and cognitive performance in a group of elderly patients whose unexplained gait instability, ventriculomegaly and disproportionately narrow sulci made them candidates for the diagnosis of NPH. The findings indicate that radiographically detectable hippocampal atrophy correlates strongly with the presence of severe cognitive symptoms in these patients. The possibility that hippocampal atrophy may be a specific marker for the pathology of Alzheimer's disease in patients with suspected NPH should be the focus of future investigations.

\section{Methods}

During a 15 month period, 16 elderly patients with a diagnosis of possible NPH were evaluated at the New York University Aging and Dementia Research Center (NYU-ADRC). These patients (aged 78-87) were selected consecutively on referral from neurologists and neurosurgeons or on presentation to the NYU-ADRC for the evaluation of cognitive complaints. All patients evidenced prominent signs of dyspraxic gait instability, symptoms of urinary incontinence or urgency, and an MRI or CT scan revealing severe ventricular enlargement in excess of sulcal prominence, as interpreted by a neuroradiologist who was not aware of the patient's clinical condition. NPH because neurological and medical evaluations revealed no explanation for the gait dysfunction other than the observation of sulcal disproportionate ventriculomegaly. These patients received a diagnosis of possible 
Figure $1 T 1$ weighted coronal MR images (TR 516, TE 21) of two patients with

ventriculomegaly and unexplained prominent gait instability: $(A)$ nondemented 80-year-old female, MMSE score $=$ 29; arrow indicates the left hippocampal formation; (B) demented 87-year-old female, MMSE score = 14; hippocampal formation atrophy is evident bilaterally.
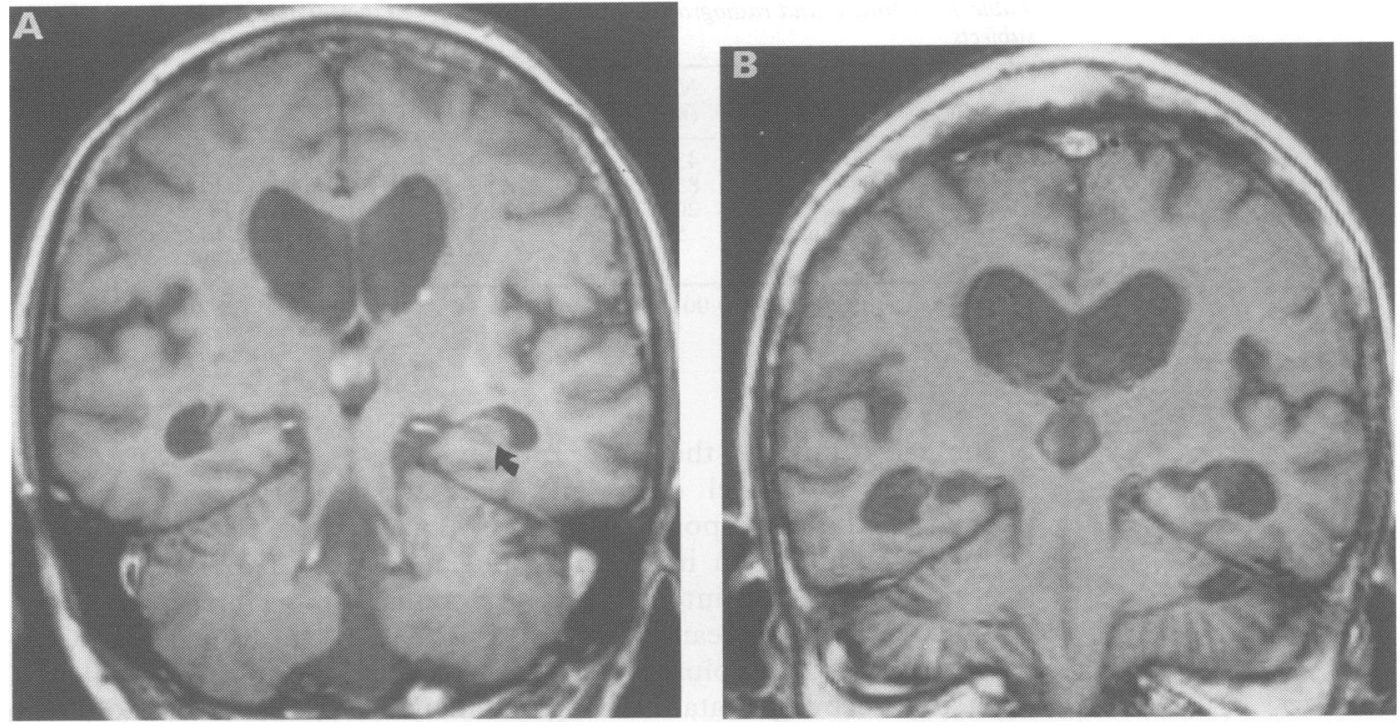

Specifically, the gait impairment could not be attributed to rheumatological disease, peripheral neuropathy, myelopathy, stroke, Binswanger's disease, Parkinson's disease, cerebellar disease, or intracranial mass in any patient.

All patients had progressive gait dysfunction for over one year and none had symptoms of significant cognitive dysfunction before the onset of the gait difficulty. Ventriculomegaly could not be attributed to a prior history of stroke, intracranial haemorrhage, meningitis, or head injury in any of the patients. Of the patients, 15 consented to indium-111 lumbar cisternography and, in each case, a pattern of radiolabelled isotope reflux into the lateral ventricles which persisted for 48 hours was detected. (The MRI scan for the one patient who did not consent to cisternography is presented in fig 1B.)

For reference purposes, the authors studied all neurologically and cognitively normal individuals (global deterioration scale $^{9} \leqslant 2$ ) between the ages of 78 and $87(n=14)$ who participated in a study of normal ageing at the NYU-ADRC between July 1988 and April 1991.

All subjects received MRI scans and were administered the mini-mental status examination (MMSE). The MMSE is a 30 point psychological scale for assessing severe cognitive impairment; a score of 23 or below has been considered diagnostic of dementia. ${ }^{10}$ Although cognitive dissimilarities have been described for NPH and Alzheimer's disease patients, ${ }^{1}$ it is uncertain whether psychological assessments can effectively distinguish the two. ${ }^{11}$ As there is no neuropsychological instrument specific for the dementia of NPH available, and because it is suspected that many demented elderly patients meeting conventional criteria for the diagnosis of NPH may have Alzheimer's disease, the MMSE satisfies the requirements of this study by providing a general means for identifying individuals with and without serious cognitive deficits.

Using a 1.5 tesla Philips Gyroscan (Shelton, Connecticut, USA), two MRI sequences were obtained: (a) a set of axial, $10 \mathrm{~mm}$ thick, contiguous, inversion, recovery images $(23 \mathrm{~cm}$ field of view (FOV), $5000 / 100 / 490 / 1 \quad(T R / T E / T I /$ excitations $)$, acquisition matrix $128 \times 64)$ covering the intracranial cavity from base to vertex; (b) a set of coronal, $4 \mathrm{~mm}$ thick, contiguous, $\mathrm{T} 1$ weighted, spin echo images $(23 \mathrm{~cm}$ FOV, $516 / 21 / 2$ (TR/TE/excitations), acquisition matrix $204 \times 256$ ) through the temporal lobe and angled $90^{\circ}$ to the long axis of the hippocampus, as determined on a $\mathrm{T} 1$ weighted sagittal scout image. The NPH patients received both sequences and the control subjects received the $T 1$ sequences only.

The modulus images from the axial inversion recovery sequence were used to measure ventricular and extraventricular CSF volumes. These images effectively suppressed signal from all intracranial tissue except CSF (CSF:brain contrast $=36: 1$ ). Using proprietary region analysis software and a mousedriven cursor, the authors defined the intracranial margin and separated ventricular from extraventricular CSF on each slice. In addition, regions containing pure (non-partial volumed) CSF were drawn at the centres of the lateral ventricles. Using a modification of the technique originally proposed by Condon et $a l,,^{12}$ the summed total signal intensity for the ventricular and extraventricular regions was then divided by the mean signal intensity of the pixels containing pure CSF to calculate total CSF volumes for both compartments. This technique is insensitive to partial volume related sources of error and permits volume recovery accuracies in the range $3-5 \% .{ }^{13}$

The coronal $\mathrm{T} 1$ slices depicting the body of the hippocampus from just behind the hippocampal head to the level of the posterior pulvinar were used to measure the size of the hippocampus. For each slice, the right and left hippocampal formation (HF) was outlined using anatomical boundaries consistent with those used by Jack et $a l^{7}$ (Ammon's horn, subiculum, alveus, fimbria). The intracranial supratentorial volume (SV) was defined on each slice by tracing the inner table 
Table 1 Clinical and radiographic characteristics of subjects

\begin{tabular}{lll}
\hline Characteristic & $\begin{array}{l}\text { NPH } \\
(n=16)\end{array}$ & $\begin{array}{l}\text { Control } \\
(n=14)\end{array}$ \\
\hline Male gender (\%) & 43.8 & 42.9 \\
Age (years) & $83.5(2 \cdot 9)$ & $82.0(2 \cdot 8)$ \\
MMSE score & $20.94(6.52)$ & $28.71(1 \cdot 20)^{\star}$ \\
Normalised hippocampal & $5.80(1.08)$ & $7.35(0.09)^{\star}$ \\
$\quad$ size $\times 10^{3}$ & &
\end{tabular}

Values are mean(SD). ${ }^{\star} p<0.001$

of the calvarium and the tentorium cerebelli. The mean HF pixel count per slice was divided by the corresponding mean SV pixel count to compute an index of hippocampal size normalised for intersubject intracranial volume variability. Mean SV was also used to normalise the CSF volumes derived from the inversion recovery data. All region analysis was performed retrospectively without knowledge of the subject's clinical status.

\section{Results}

As there were no significant differences between right and left hippocampal sizes ( $t=$ $0.36, p>0.80$ ), the mean value for both hemispheres combined is reported. Table 1 illustrates the sex, age, MMSE score, and hippocampal size relationships among the $16 \mathrm{NPH}$ patients and the 14 controls. As a group, NPH patients had lower MMSE scores and smaller normalised hippocampal formation sizes than controls. Figure 2 demonstrates the relationship between normalised hippocampal size (HF/SV) and MMSE score for the entire sample. It can be appreciated that hippocampi of NPH patients overlap in size with those of normal controls only for individuals with high MMSE scores. As suggested by this plot, normalised hippocampal size in the $16 \mathrm{NPH}$ patients correlated strongly with MMSE score $(r=0.75, p$ $<0.001)$. Correlations among MMSE score and normalised ventricular CSF volume $(r=$ $0.01, p=0.97)$, normalised extraventricular CSF volume $(r=0.01, p=0.96)$, and extraventricular:ventricular CSF ratio $(r=0 \cdot 19, p$ $=0.49$ ) were not significant. There was no significant correlation between the normalised measures of ventricular CSF and hippocampal size $(r=0.20, p>0.47)$.

Figure 2 Relationship of MMSE score to normalised hippocampal size $(H F / S V) \times 10^{3}$ for NPH patients and cognitively normal controls. Regression line is based on NPH data only; - NPH; O, control.

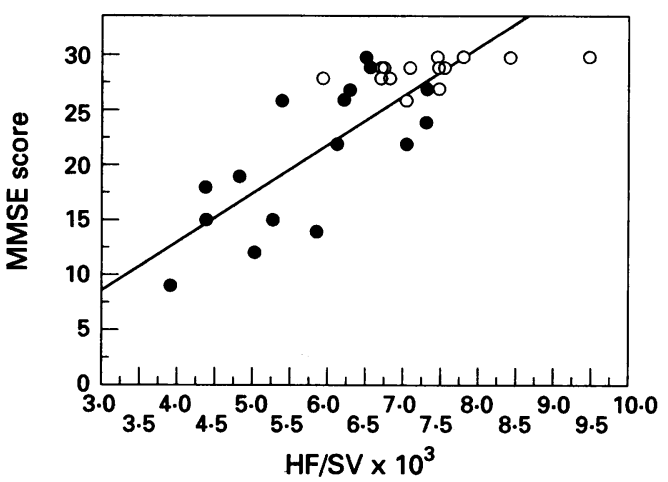

Table 2 Radiographic characteristics of NPH patients

\begin{tabular}{llll}
\hline & $\begin{array}{c}M M S E \\
\leqslant 23 \\
(n=9)\end{array}$ & $\begin{array}{l}M M S E \\
>23 \\
(n=7)\end{array}$ & $p$ \\
\hline $\begin{array}{l}\text { Age (years) } \\
\begin{array}{l}\text { Normalised hippocampal } \\
\text { size } \times 10^{3}\end{array}\end{array}$ & $\begin{array}{c}82.9(3.3) \\
5.22(1.00)\end{array}$ & $\begin{array}{l}84.3(2.4) \\
6.54(0.67)\end{array}$ & $\begin{array}{l}0.36 \\
\text { Normalised ventricular } \\
\quad \text { CSF volume }\end{array}$ \\
$\begin{array}{l}\text { Normalised extraventricular } \\
\text { CSF volume }\end{array}$ & $0.88(0.38)$ & $0.94(0.20)$ & 0.71 \\
$\begin{array}{l}\text { Extraventricular: } \\
\text { ventricular }\end{array}$ & $2.12(0.94)$ & $2.21(0.45)$ & 0.81 \\
CSF ratio & $2.20(1.04)$ & $2.44(0.71)$ & 0.61 \\
\hline Values are mean & & & \\
\hline
\end{tabular}

Table 2 demonstrates differences in hippocampal size, ventricular CSF volume, extraventricular CSF volume, and extraventricular:ventricular CSF ratios for $\mathrm{NPH}$ patients with and without dementia as defined by an MMSE score $\leqslant 23$. Of the four brain characteristics examined, significant differences were seen only for the normalised measure of hippocampal size.

\section{Discussion}

In this report the authors have demonstrated an association between hippocampal size and severe cognitive dysfunction in a sample of elderly patients with sulcal disproportionate ventriculomegaly and gait impairment. As no other explanation for the gait impairment could be identified, NPH was considered a plausible diagnosis. Although brain measures of hydrocephalic severity such as ventricular volume and extraventricular:intraventricular CSF ratio were also examined, these were not found to correlate with cognitive performance. Pathological changes affecting the hippocampus may explain the amnesic deficits exhibited by patients with Alzheimer's disease and other disorders. ${ }^{14} 15$ The present finding that hippocampal atrophy is associated with severe cognitive impairment in patients suspected of having NPH suggests an explanation for the dementia of some individuals with this diagnosis.

Proposals concerning the pathogenesis of hippocampal atrophy in NPH remain speculative. Although the mechanical effects of chronic ventricular dilatation cannot be excluded as an aetiological factor, the authors propose that hippocampal atrophy in elderly NPH patients may represent the concomitant presence of an independent neurodegenerative process such as Alzheimer's disease. This hypothesis is based on the following considerations: (a) recent radiographic reports demonstrate a strong association between Alzheimer's disease and hippocampal atrophy ${ }^{6}$; (b) little evidence was observed for hippocampal atrophy in patients with prominent gait disturbance, severe ventriculomegaly, and an absence of significant cognitive deficitssuch patients are believed to be unlikely to have Alzheimer's disease ${ }^{1}$; (c) the present data reveal that hippocampal atrophy is not an invariable feature of severe ventriculomegaly; (d) the very high prevalence of Alzheimer's 
disease as a cause of dementia in the elderly makes it probable that many of the demented patients in the present sample could be suffering from this diagnosis.

It is recognised that elderly patients with dementia and gait impairment may have Alzheimer's disease even if they also meet conventional radiographic criteria for the diagnosis of NPH. ${ }^{1-4}$ As NPH patients with concomitant pathology of Alzheimer's disease may respond less favourably to ventricular shunting than patients with "pure" $\mathrm{NPH}$, a technique for accurately distinguishing between these two conditions could significantly facilitate clinical decision making. Although postmortem validation is needed to determine whether hippocampal size measurements may provide such a technique, the hypothesis that hippocampal atrophy can identify the presence of Alzheimer's disease in patients suspected of NPH may have implications that are amenable to clinical investigation. For example, such atrophy should be rare in younger NPH patients who are at less risk for Alzheimer's disease. Furthermore, many NPH patients are believed to manifest a "subcortical" profile of psychological abnormalities characterised by mental slowing and apathy, rather than the more "cortical" deficits of aphasia and dyspraxia commonly noted in Alzheimer's disease. ${ }^{1}$ NPH patients with hippocampal atrophy might therefore tend to exhibit features of "cortical dementia", whereas patients without such atrophy may more closely resemble the "subcortical" pattern. Finally, shunt outcome studies would be expected to reveal a poorer response for patients with hippocampal atrophy. Future investigations examining these and other issues will help determine the clinical use of hippocampal size measurements in the evaluation of patients with NPH.

This work was supported in part by NIH grants IP30AG08051, IP30MH43486, IR01MH43965, and IR01AG03051. The authors also acknowledge generous supIR01AG03051. The authors also acknowledge generous support from the Orentreich Foundation for the Advancement of
Science, (Cold Spring, NY) and are indebted to Martha Science, (Cold Spring, NY) and are indebted to Martha
Helmers and Anthony Jalandoni for their expert assistance in Helmers and Anthony
preparing the figures.

1 Katzman R. Normal pressure hydrocephalus. In: Wells C, ed. Dementia. Philadelphia: FA Davis Co. 1977:69-92.

2 Sohn RS, Siegel BA, Gado M, Torack RM. Alzheimer's disease with abnormal cerebrospinal fluid flow. Neurology 1973;23:1058-65.

3 Coblentz JM, Mattis S, Zingesser LH, Kasoff SS, Wisniewski HM, Katzman R. Presenile dementia. Clinical aspects and evaluation of cerebrospinal fluid dynamics. Arch Neurol 1973;29:299-308.

4 Vassilouthis J. The syndrome of normal-pressure hydrocephalus. $\mathcal{f}$. Teurosurg 1984;61:501-14.

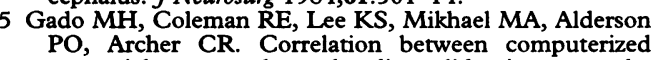
transaxial tomography and radionuclide cisternography in dementia. Neurology 1976;26:555-60.

6 de Leon MJ, George AE, Stylopoulos LA, Smith G, Miller DC. Early marker for Alzheimer's disease: The atrophic hippocampus. Lancet 1989;ii:672-3.

7 Jack CR Jr, Petersen RC, O'Brien PC, Tangalos EG. MRbased hippocampal volumetry in the diagnosis of Alzheimer's disease. Neurology 1992;42:183-8.

8 Fisher CM. Hydrocephalus as a cause of disturbances of gait in the elderly. Neurology 1982;32:1358-63.

9 Reisberg B, Ferris SH, de Leon MJ, Crook T. The Global Deterioration Scale (GDS). Psychopharmacol Bull 1988;24:661-3.

10 Cockrell JR, Folstein MF. Mini-mental state examination (MMSE). Psychopharmacol Bull 1988;24:689-92.

11 Adams RD, Victor M. Principles of Neurology. New York: McGraw-Hill 1989:508.

12 Condon BR, Patterson J, Wyper D, et al. A quantitative index of ventricular and extraventricular intracranial CSF volumes using MR imaging. F Computer Assist Tomogr 1986;10:784-92.

13 Chandra $R$, Rusinek $H$. Segmentation of brain tissue from magnetic resonance images. Medical Imaging V: Image Processing. SPIE Biomed Image Process 1991;1445: 133-44.

14 Zola-Morgan S, Squire LR, Amaral DG. Human amnesia and the medial temporal region: Enduring memory impairment following a bilateral lesion limited to field CA1 of the hippocampus. F Neurosci 1986;6:2950-67.

15 Hyman BT, Van Hoesen GW, Damasio AR. Memoryrelated neural systems in Alzheimer's disease: an related neural systems in Alzheimer's dise 